\title{
Books received
}

\section{An Asperger Marriage}

By Gisela \& Christopher Slater-Walker. London: Jessica Kingsley. 2002. 160 pp EI2.95 (pb). ISBN I 843100177

\section{Being Alive. Building} on the work of Anne Alvarez

Edited by Judith Edwards. Hove: Brunner-Routledge. 2001. 239 pp. EI6.99 (pb). ISBN I 5839| I3। 6

\section{Beyond Madness. Psychosocial} Interventions in Psychosis

Edited by Joseph H. Berke, Margaret Fagan, George Mak-Pearce \& Stella Pierides-Müller. London: Jessica Kingsley. 2002. 240 pp EI7.95 (pb). ISBN I 85302889

\section{Bullying in Adulthood. Assessing the Bullies and their Victims}

By Peter Randall. Hove: Brunner-Routledge. 200I. 223 pp. $€ I 5.99$ (pb). ISBN $04 I 5236940$

\section{Culture, Health and Illness (4th edn)}

By Cecil G. Helman. London: Arnold. 2000. 336 pp. $€ 19.99$ (pb). ISBN 0750647868

\section{Current Treatments} of Obsessive-Compulsive Disorder

Edited by Michele Tor tora Pato \& Joseph

Zohar. Washington, DC: American

Psychiatric Press. 200I. 265 pp. $€ 41.95$ (hb).

ISBN 0880487798

\section{Essentials of Clinical \\ Psychopharmacology}

Edited by Alan F. Schatzberg \& Charles B. Nemeroff. Washington, DC: American Psychiatric Press. 2001.799 pp. $€ 63.50$ (pb). ISBN I 585620173

Family Therapy and Mental Health. Innovations in Theory and Practice

Edited by Malcolm M. MacFarlane.

Binghamton: The Haworth Press. 200I.

440 pp. $€ 48.30$ (hb), $€ 27.60$ (pb).

ISBN 0789008807 (hb), 0789015897 (pb)
Group Action. The Dynamics of Groups in Therapeutic, Educational and Corporate Settings

ByT. Martin Ringer. London: Jessica Kingsley. 200I. 304 pp. $€ \mid 9.95$ (pb). ISBN I 843100282

\section{Hypochondriasis. Modern} Perspectives on an Ancient Malady

Edited by Vladan Starcevic \& Don R. Lipsitt. Oxford: Oxford University Press. 2001. 416 pp. $€ 42.50$ (hb). ISBN 0195126769

\section{Juvenile Sexual Homicide}

By Wade C. Myers. London: Academic Press. 2002. 198 pp. $€ 53.95$ (hb). ISBN 0125123035

Music Therapy, Sensory Integration and the Autistic Child

By Dorita S. Berger. London: Jessica Kingsley. 200I. 256 pp. $€ I 5.95$ (pb). ISBN I I843I0 7007

\section{Neuropsychiatry: An Introductory Approach}

By David B. Arciniegas \& Thomas P. Beresford. Cambridge: Cambridge University Press. 2001.459 pp. $€ 34.95$ (pb). ISBN 0521644313

Obsessive-Compulsive Disorder. Theory, Research and Treatment Edited by Richard P. Swinson, Martin M. Antony, S. Rachman \& Margaret A. Richter. London: Guilford Press. 1998. 494 pp. ŁI 9.95 (pb). ISBN I 572307323

\section{Psychiatry in Canada: 50 years (195I to 200I)}

Edited by Quentin Rae-Grant. Ottawa: Canadian Psychiatric Association. 2001. 320 pp.CAN\$16.00 (pb). ISBN 0969999275
Quality of Life in Child and Adolescent IIIness. Concepts, Methods and Findings

Edited by Hans M. Koot \& Jan L.Wallander New York: Taylor \& Francis. 200I. 479 pp. $€ 24.95$ (pb). ISBN I 5839I 2347

Schizophrenia and Comorbid Conditions. Diagnosis and Treatment

Edited by Michael Y. Hwang \& Paul.C. Bermanzohn. Washington, DC: American Psychiatric Press. 2001.253 pp. $€ 38.95$ (hb). ISBN $08804877 \mid 2$

Terror and Transformation. The Ambiguity of Religion in Psychoanalytic Perspective

By J.W. Jones. London: Taylor and Francis. 2002. I38 pp. $€ \mid 2.99$ (pb). ISBN I 5839I 1936

Thalamocortical Assemblies. How Ion Channels, Single Neurons and Large-Scale Networks Organize Sleep Oscillations

By Alain Destexhe \& Terrence J. Sejnowski. Oxford: Oxford University Press. 2001. 47 I pp. $€ 85.00$ (hb). ISBN 0198524250

Traumatic Pasts. History, Psychiatry, and Trauma in the Modern Age, 1870-1930

Edited by Mark S. Micale \& Paul Lerner. Cambridge: Cambridge University Press. 200I. 33I pp. $€ 40.00$ (hb). ISBN 0521583659

Undertaker of the Mind. John Monro and Mad-Doctoring in Eighteenth-Century England

Jonathan Andrews \& Andrew Scull. Berkeley, London: University of California Press. 2001. 386 pp. $€ 24.95$ (hb). ISBN 05202315 I । 